\title{
Payment by results in mental health: the current state of play in England ${ }^{\dagger}$
}

\author{
Andrew Fairbairn
}

Abstract The Department of Health is introducing a 'payment by results' system for mental health services in the UK. The system is already being used in the acute healthcare sector, but is still under development for application in mental healthcare. This article outlines the concept of payment by results, briefly reports on its use in other countries and describes the pilot study underway in England to define currencies to be used in a payment by results system planned for NHS mental healthcare throughout England and Wales.

'Payment by results' is a method of paying for the services of hospitals and other providers. Plans are well underway to use it within the National Health Service (NHS) in England, and some health authorities began to introduce it in 2005. The discussion of payment by results is usually peppered with abbreviations. I have used these only rarely in this article, but a list of those most commonly encountered in the literature appears in Box 1.

Payment by results has three central components. The first is activity-based funding, which contrasts with the more traditional block contract agreements. For service providers, more work will generate more income or, indeed, less work will generate less.

Second, the amount of work tends to be measured in the context of healthcare resource groups. These are groupings of individual cases that are sufficiently clinically similar and require similar treatment resources. In the acute healthcare sector they tend to be based on international categories of diagnosis. They also take into account procedures, complications and co-existing illnesses. Therefore any payment by results system seeks to provide a coherent manageable way of classifying the mixed cases treated within a hospital. This is why payment by results is sometimes called a system of case-mixed funding.

Third, payment is made according to a national tariff. This tariff is a price tag for any individual healthcare resource group. The price tends to be based on the average cost of treatment across the NHS. This

${ }^{+}$For a commentary on this article see pp. 7-9, this issue.

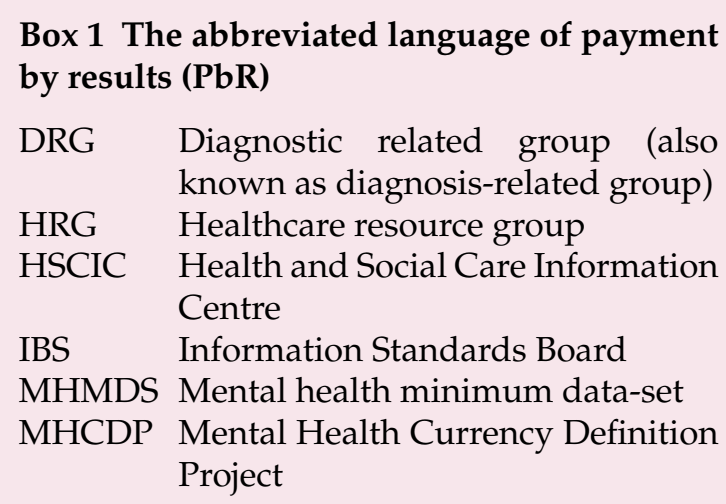

Box 1 The abbreviated language of payment by results (PbR)

DRG Diagnostic related group (also known as diagnosis-related group)

HRG Healthcare resource group

HSCIC Health and Social Care Information Centre

IBS Information Standards Board

MHMDS Mental health minimum data-set

MHCDP Mental Health Currency Definition Project

national tariff means that contractual negotiations between commissioners and providers no longer require price negotiation but can concentrate on quantity and quality of care.

It is important to recognise that a number of countries have investigated the possibility of a payment by results system and none so far has successfully implemented such a scheme. Some results of studies in Australia and New Zealand are briefly discussed below but one must draw the conclusion that there maybe a message in this. Nevertheless, the system has been introduced in most of the acute sector of the NHS in England and the Department of Health has put pressure on service commissioners to use it. There is anecdotal evidence that, as the system has not yet been implemented in the mental health sector, commissioners have taken the opportunity to squeeze mental health service funding in order to meet cost pressures in acute (non-psychiatric)

Andrew Fairbairn is a consultant old age psychiatrist (St Nicholas Hospital, Gosforth, Newcastle upon Tyne NE3 3XT, UK. Email: andrew.fairbairn@nmht.nhs.uk) and a former Registrar of the Royal College of Psychiatrists. He has a special interest in health service management. 
services. Therefore, despite reservations, the payment by results system is probably one that mental health services should join if a viable system can be introduced.

\section{The rationale behind payment by results in the NHS}

The Department of Health believes that payment by results will encourage providers to increase the level of their activity and improve their efficiency. It should provide an incentive for commissioners to seek out lower-cost forms of care in order to reuse the money saved. Payment by results requires an improvement in information systems, which should lead to wider benefits. In addition, services should become more personalised and responsive.

\section{International use of the system}

The current NHS project built on preparatory work undertaken in the UK in 1999-2000 and on the project methodology of the New Zealand Mental Health Classification and Outcomes Study (Gaines et al, 2003). Similar work was undertaken in Australia (Buckingham et al, 1998).

This work found that case-mix classification had the potential to be used in specialist mental health services to improve routine data collection and inform management and planning decisions. It could help to explain the variation between providers, create a profile of the treated population and benchmark services, as well as inform funding. Neither New Zealand or Australia has yet formally used their case-mix groups for the latter purpose.

The Australian and New Zealand models do not directly reflect service patterns and patient profiles in the NHS. Consequently, the Casemix Service (http://www.ic.nhs.uk/casemix) is delivering a bespoke currency classification system (see below), which will endeavour to incorporate best practice of existing international systems.

\section{Payment by results in England}

A pilot is underway in England to develop clinically meaningful and appropriate classifications ('currencies') to accurately describe mental healthcare received by adults of working age and older.

Preliminary findings from the first stage of the pilot (data collection) may be available by the end of 2006. The second stage (data proving) will begin in April 2007. Final results will be available early in 2007. Further details follow in the next section.
Irrespective of payment by results policy, the majority of mental health service providers advocate the production of high-quality information that can be used to optimise service performance in the light of patients' expectations and needs, and to monitor treatment outcomes.

Payment by results policy is focusing attention on an increased understanding of service delivery, linked to financial reward. The Department of Health believes that the ability of providers to better understand their services will support future NHS practice and development, and underpin the creation of a patient-led NHS.

According to the Sainsbury Centre for Mental Health (2004: p. 4), advantages are expected to include, but are not limited to, the following:

- mental health service providers will be paid for the work they undertake, and will not suffer undue reductions in income as a result of acute-sector financial pressure on overall resources

- more accurate information about what is done for a patient should furnish better patient care over time, as outcome measures will be able to judge what works best

- increased understanding of care provision will enable appropriate clinical comparison

- audit of the system will improve overall patient care.

My worries in relation to disadvantages are mainly about misfunding or underfunding:

- providers are paid exactly the same for every case in a given healthcare resource group, so treating only the 'easier' patients within that group should reduce costs

- providers might also try to skimp on the quality of care in order to reduce costs; a typical example of this would be to discharge patients prematurely

- providers might try to manipulate the information so that patients are classified in more costly healthcare resource groups.

Payment by results is expected to address existing issues by:

- enabling stronger, integrated local health and social care commissioning, at primary care trust or practice level

- supporting best practice

- 'unbundling' from existing block contracts, which should introduce choice and plurality, and move treatments to the community or primary care

- allowing direct payments and individual budgets for social care elements where necessary. 


\section{The Mental Health Currency Definition Project}

As the first stage of setting up a payment by results system for mental healthcare, the Department of Health has commissioned the Health and Social Care Information Centre (HSCIC) to develop 'currencies' for mental health services.

Currencies are standard groupings of clinically similar treatments or similar client groups that use common levels of healthcare resource. They can therefore be used within the health service for performance management and inter- and intraservice comparison. They may also be used for costing and reimbursement purposes.

The three stages of the currency definition project are outlined in Fig. 1.

\section{The scope of the project}

It has been agreed that the currency definition project will begin by focusing on in-patient and communitybased mental health services for adults of working age and older people in England, although a variety of different hypotheses are being tested on various sets and subsets of the data.

Services provided through child and adolescent mental health services, learning disability (intellectual disability) services, substance misuse services, forensic services, secure units and services for patients receiving exclusively either social care or primary care are at present beyond the scope of the project.

\section{Pilot study - phase one, stage one}

The project is at the first stage of a phased approach to developing currencies that can accurately describe the patient and the resource implications of treating that patient in clinical terms.

A review of data sources revealed that there was no repository available to use for retrospective

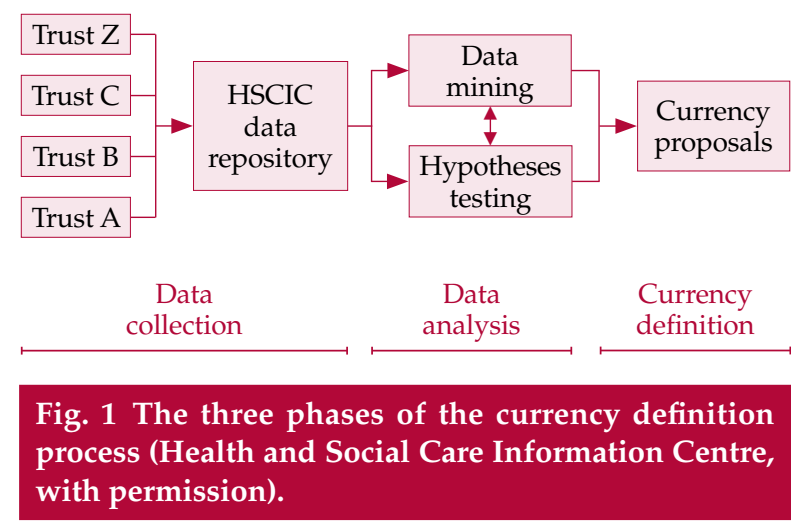

analysis to generate the currencies. Therefore a data collection programme was established to provide a comprehensive set of patient-level data.

Pilot sites in England are focusing on data collection, and the project as a whole is looking to maximise the sample size, quality and uniformity of the data collected. This will allow a more effective analysis to support the identification of appropriate currencies for mental health.

Once sufficient data have been received, analysis will begin. As an initial step towards creating a currency it will take two approaches: data mining and the testing of existing hypotheses (e.g. related to the use of care pathways). An example of a hypothesis being tested is that resource utilisation in the treatment of a particular patient over a certain period can be predicted by using non-identifiable patient data in the following combinations:

- age and rating on items 1, 2, 3 and 12 of the Health of the Nation Outcomes Scales (HoNOS; http://www.rcpsych.ac.uk/crtu/healthofthe nation.aspx)

- ethnicity

- diagnosis

- care programme approach (CPA) level, setting and team type

- age, ethnicity, gender and legal status

- cluster assignment.

The standard data-set being collected by the majority of trusts is shown in Fig. 2. However, variations between trusts do exist because of local circumstances, and the data-set being collected by the mental health trusts in the north of England, which are following an approach similar to that shown in Fig. 2, with the exception of the following:

- HoNOS Plus ratings are being collected rather than HoNOS

- additional cluster information is being collected.

\section{The next stages}

Once analysis is complete, proposed currencies will be tested by a group of trusts to validate them as useable before they are presented for approval by the Expert Working Group and the Project Board of the MHCDP.

Approved final currencies will be passed to the Department of Health after the pilot phase described above.

It is expected that the currencies for mental health services will continue to evolve over time, to better reflect changing clinical practice and mental health service redesign, as well as to incorporate new services within the sphere of payment by results. 


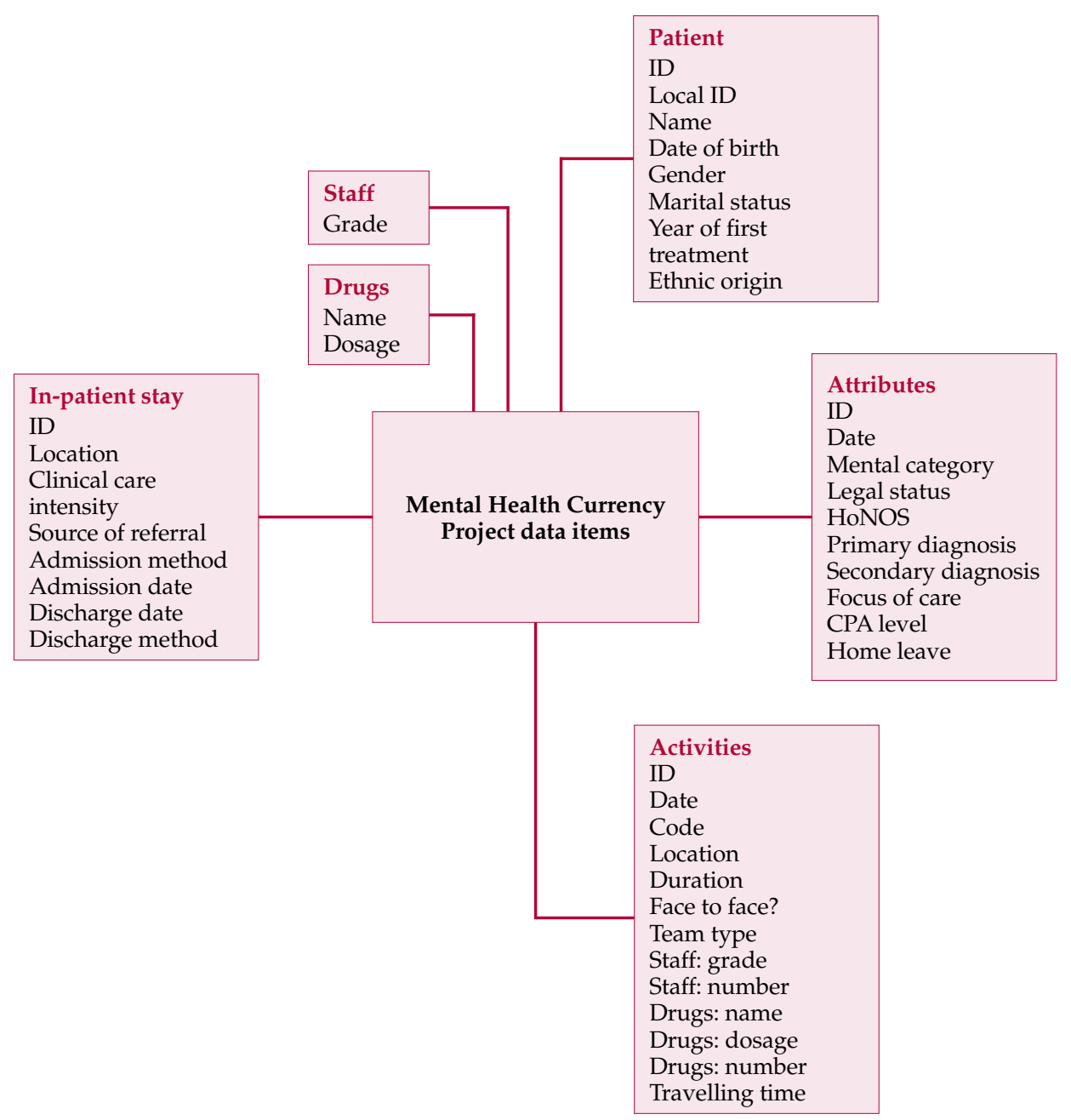

Fig. 2 Data collection for the Mental Health Currency Definition Project. The type of data appears in red lettering, and the data elements in black (Health and Social Care Information Centre, with permission).

\section{Conclusions}

Anticipating the MHCDP's report on payment by results in mental healthcare, it would appear that any solution will be radically different from the traditional healthcare resource grouping used in the acute (non-psychiatric) sectors of the health economy. It is also obvious there are no quick and easy answers, so the anticipated pilot phase will involve much refinement and probably still leave some unanswered issues.

\section{Declaration of interest}

None.

\section{References}

Buckingham, W., Burgess, P., Solaman, S., et al (1998) Developing a Casemix Classification for Mental Health Services. Vol. 1: Main Report. Commonwealth Department of Health.

Gaines, P., Bower, A., Buckingham, W., et al (2003) New Zealand Health Classification and Outcomes Study: Final Report. Health Research Council of New Zealand.

Sainsbury Centre for Mental Health (2004) Policy Paper 4: Payment by Results: What Does it Mean for Mental Health? SCMH. 